\title{
Management of a duplicated inferior vena cava in thoracoabdominal aortic aneurysm repair
}

\author{
Melissa M. Levack, MD, ${ }^{\mathrm{a}}$ Suresh Keshavamurthy, MD, ${ }^{\mathrm{a}}$ Paul Schoenhagen, MD, ${ }^{\mathrm{b}}$ and \\ Douglas R. Johnston, MD, ${ }^{\text {a }}$ Cleveland, Ohio \\ From the Departments of ${ }^{\mathrm{a}}$ Thoracic and Cardiovascular Surgery and ${ }^{\mathrm{b}}$ Radiology, Heart and Vascular Institute, \\ Cleveland Clinic, Cleveland, Ohio. \\ Received for publication Aug 2, 2016; revisions received Oct 5, 2016; accepted for publication Oct 13, 2016; \\ available ahead of print Dec 2, 2016. \\ Copyright (C) 2016 by The American Association for Thoracic Surgery
}

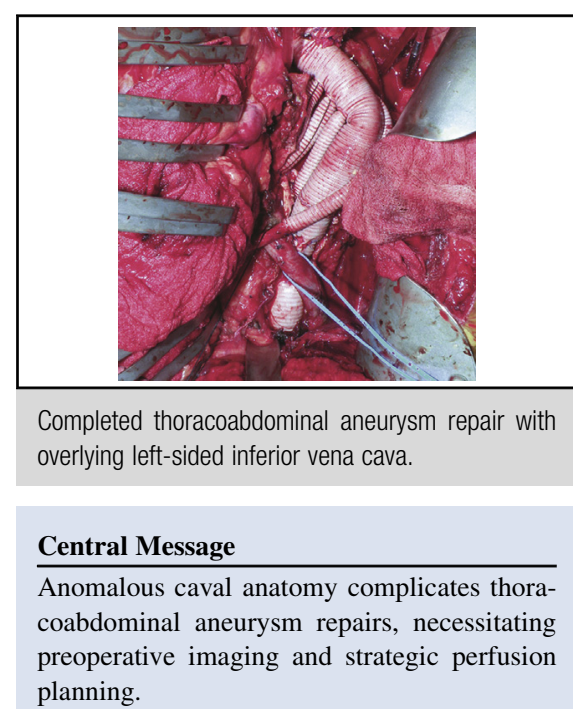

See Editorial Commentary page e43.
Venous anomalies in association with aortoiliac disease, including duplicate inferior vena cava (IVC) and left IVC, have been described ${ }^{1,2}$; however, surgical management of thoracoabdominal aneurysms (TAAs) presents unique challenges in the setting of aberrant anatomy. One description of a TAA repair with a clamp-and-sew technique in the setting of a left IVC has been reported. There are currently no reports of TAA repair after thoracic endovascular aortic repair with left heart bypass. We present the case of a patient with a duplicated IVC and describe the operative and perfusion strategies for TAA repair.

\section{CLINICAL SUMMARY}

A 61-year-old man underwent a type A dissection repair with hemiarch in 1995, followed in 2006 by thoracic endovascular aortic aneurysm repair for his residual type $\mathrm{B}$ dissection with aneurysmal degeneration. When he was later referred to our institution, the proximal end of the stent graft excluded the false lumen but the distal end of the stent graft failed to appose the aortic wall. Beyond the stent, there was an $8.5-\mathrm{cm}$ dilation of the diaphragmatic segment, with the dissection flap extending through the abdominal aorta to both common iliacs. In addition, the patient was noted to have a duplicated IVC (Figure 1). The aorta was exposed through a left thoracoabdominal extraperitoneal approach, and the duplicated IVC was isolated. Left heart bypass was established by cannulation of the left inferior pulmonary vein and the left femoral artery. The patient was cooled to $32^{\circ} \mathrm{C}$. The aorta was crossclamped in the retrocardiac and supraceliac locations and incised. One distal stent ring was removed, and a 24-mm Gelweave

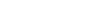

4-branch graft (Vascutek Ltd a Terumo Company, Glasgow, UK) was sewn to it with 2 layers of felt incorporating the wall of the aorta. (Figure 2, A). The branch graft was clamped distally, and the proximal aorta was closed around the stent graft. The distal aorta was opened to the bifurcation, and the visceral vessels were perfused with cold blood. Femoral flow was ceased, and an extensive fenestration and endarterectomy procedure was performed because of severe calcification of the distal aorta. A 24-mm graft was sewn to the distal aorta and then clamped to resume femoral circulation. Individual grafts were sewn to the right renal, superior mesenteric, celiac, and left renal arteries. The distal graft was passed under the left IVC in anatomic position, sewn end to end to the proximal graft, and pleated to reduce its diameter and impingement on the left IVC (Figure 2, B). The patient was rewarmed, weaned from bypass, and closed in standard fashion. He awoke neurologically intact and was extubated on postoperative day 1 . His creatinine remained normal. The patient's recovery was notable for paroxysmal atrial fibrillation necessitating digoxin and warfarin and for difficult-to-manage pain. He was discharged on postoperative day 12. At his 3-month follow-up, the patient continues to do well. 

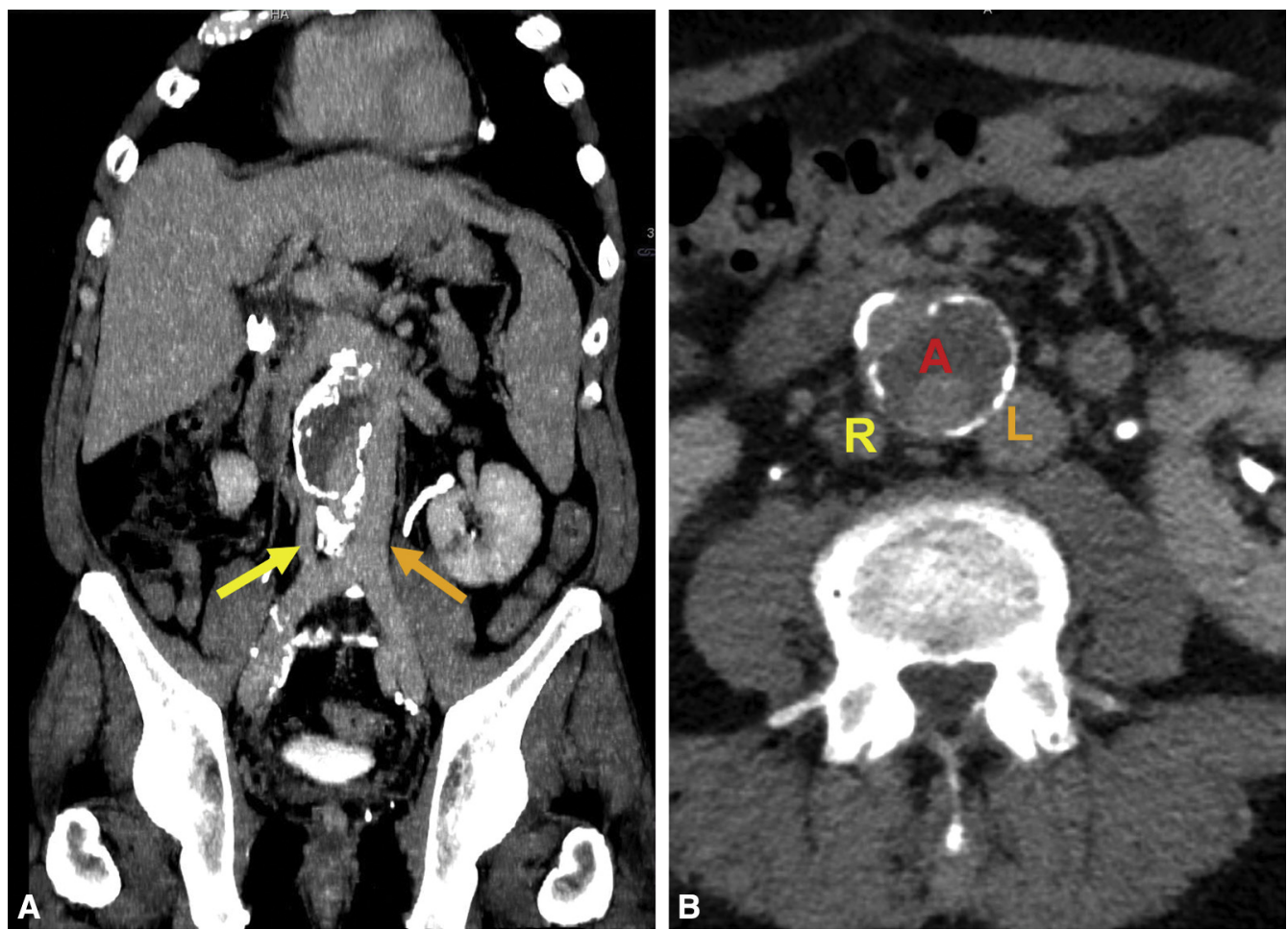

FIGURE 1. Preoperative coronal (A) and axial (B) computed tomographic imaging depicting the anatomic relationship of the smaller right-sided (yellow) and larger left-sided (orange) inferior venae cavae to the aorta.

\section{DISCUSSION}

The IVC is formed from the subcarinal venous system, which is made up of paired veins on both sides of the aorta that commonly anastomose at the level of the left renal vein. The left subcardinal vein regresses during normal embryologic development.
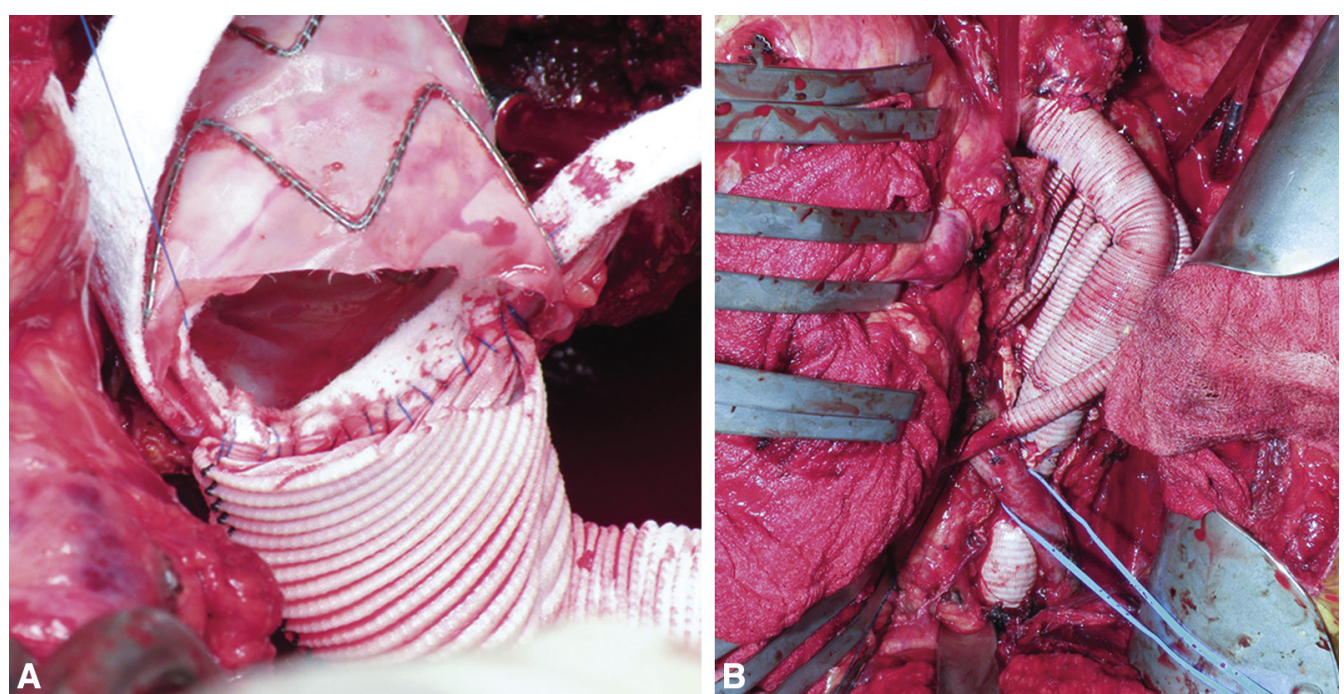

FIGURE 2. Intraoperative photographs demonstrating the felt sandwich used to reinforce the anastomosis between the previously placed endovascular stent graft and the new branch graft during repair (A) and depicting the accessory left IVC (marked by the blue vessel loop) crossing over the aortic graft after completed reconstruction (B). 
Incomplete regression can lead to a duplicate IVC, which occurs in $0.3 \%$ to $0.4 \%$ of the population, whereas regression of the right subcardinal vein leads to a left-sided IVC, which has an incidence of $0.2 \%$ to $0.5 \%$. . $^{4,5}$ Despite the infrequency and often asymptomatic nature of these anomalies, identification of these structures is critical in aortic surgery, because left-sided caval systems can complicate dissection and exposure, increase the risk of hemorrhage, and make aortic reconstruction more challenging. Preoperative computed tomographic imaging and identification of the accessory IVC in relation to the distal aorta is critical in planning appropriate repair. We were able to dissect the duplicate IVC freely away from the aorta and to retract it laterally to allow exposure of the distal anastomosis; however, we were prepared to use other perfusion strategies if this could not be achieved safely. The left IVC could have been divided to allow better exposure, and full cardiopulmonary bypass could then have achieved by placing a venous cannula in the left IVC to allow drainage of the lower body during division of the vein. Because the right IVC was small, the left IVC would need to be reconstructed as the precise venous return of the lower body is unknown in a duplicated system.

In summary, the surgical and endovascular management of TAA has grown in recent years with the addition of techniques such as left heart bypass that help to minimize visceral, spinal, and lower extremity ischemia. Anomalous caval anatomy needs to be considered in cases of TAA repair.

\section{References}

1. Niino T, Unosawa S, Shimura K. Ruptured abdominal aortic aneurysm with left-sided inferior vena cava. Ann Vasc Surg. 2012;26: 1012.e9-11.

2. Rispoli P, Conforti M, Cassatella R, Varetto G, Melloni CD, Raso AM. Leftsided inferior vena cava in patients submitted to aorto iliac surgery. Our experience and review of the literature. J Cardiovasc Surg (Torino). 2001;42: $249-55$.

3. Giglia JS, Thompson JK. Repair of a thoracoabdominal aortic aneurysm in the presence of a left-sided inferior vena cava. J Vasc Surg. 2004;40: 161-3.

4. Ang WC, Doyle T, Stringer MD. Left-sided and duplicate inferior vena cava: a case series and review. Clin Anat. 2013;26:990-1001.

5. Aljabri B, MacDonald PS, Satin R, Stein LS, Obrand DI, Steinmetz OK. Incidence of major venous and renal anomalies relevant to aortoiliac surgery as demonstrated by computed tomography. Ann Vasc Surg. 2001;15: $615-8$. 